\title{
Clinical and Cytogenetical Characteristics-Related Nomograms for Assessing Outcome of Acute Myeloid Leukemia Patients Post IA Induction
}

Na Wang

Wenzhou Medical University First Affiliated Hospital

Kanchun Dai

University of the Chinese Academy of Sciences

Aakash Desai

University of Connecticut

Bei Ge

Wenzhou Medical University Eye Hospital

Weihong Lin

Wenzhou Medical University First Affiliated Hospital

Haige Ye ( $\nabla$ haigeyeah@sina.com )

Wenzhou Medical University First Affiliated Hospital https://orcid.org/0000-0003-2693-4568

Research article

Keywords: acute myeloid leukemia, nomogram, overall survival, disease-free survival.

Posted Date: August 26th, 2020

DOl: https://doi.org/10.21203/rs.3.rs-60451/v1

License: () (1) This work is licensed under a Creative Commons Attribution 4.0 International License. Read Full License 


\section{Abstract}

Background: Acute myeloid leukemia(AML) is a highly heterogeneous hematological malignancy. Despite, increase in treatment options for AML over the past decade, prognosis for AML remain dismal. Numerous prognostic models have been developed for this disease, however nomograms predicting longterm survival in AML patients after induction chemotherapy(IA regimen) have not been described.

Method: We constructed nomograms to predict disease-free survival(DFS) and overall survival(OS) by analyzing the cohort of patients with de novo non-M3 AML patients who underwent induction chemotherapy between June 2008 to August 2019. We utilized univariable and multivariable Cox proportional hazards regression analyses to obtain the selected variables for the nomograms. The discriminative ability and calibration were tested using $\mathrm{C}$ statistics, calibration plots, and Kaplan-Meier curves.

Results: A total of 360 patients who underwent induction chemotherapy with IA regimen were included in the study. Of these $55 \%$ were male with a median age was 48 years. Using the univariate and multivariate analyses, the following variables were identified in the prediction of DFS: age $(\mathrm{HR}, 1.770 ; 95 \% \mathrm{Cl}, 1.160$ 2.702; $\mathrm{P}=0.008), \mathrm{Hb}(\mathrm{HR}, 0.634 ; 95 \% \mathrm{Cl}, 0.462-0.870 ; \mathrm{P}=0.005)$, albumin(HR, $0.473 ; 95 \% \mathrm{Cl}, 0.363-0.615 ; \mathrm{P}$ $<0.001$ ) and cyto/molecular risk group(intermediate vs. favorable: $\mathrm{HR}, 1.614 ; 95 \% \mathrm{Cl}, 1.128-2.309 ; \mathrm{P}=$ 0.001 ; poor vs. favorable: $\mathrm{HR}, 2.459 ; 95 \% \mathrm{Cl}, 1.645-3.676 ; \mathrm{P}<0.001)$ at the time of diagnosis, and alloHSCT treatment(HR, $0.341 ; 95 \% \mathrm{Cl}, 0.234-0.497 ; \mathrm{P}<0.001)$. Factors which predicted OS were $\mathrm{Hb}(\mathrm{HR}$, $0.616 ; 95 \% \mathrm{Cl}, 0.438-0.866 ; \mathrm{P}=0.005)$, albumin $(\mathrm{HR}, 0.448 ; 95 \% \mathrm{Cl}, 0.340-0.591 ; \mathrm{P}<0.001)$, cyto/molecular risk group (intermediate vs. favorable: $\mathrm{HR}, 1.558 ; 95 \% \mathrm{Cl}, 1.068-2.275 ; \mathrm{P}=0.021$; poor vs. favorable: $\mathrm{HR}$, 2.348; 95\% Cl, 1.523-3.620; $\mathrm{P}<0.001)$, allo-HSCT treatment (HR, 0.256; 95\% Cl, 0.166-0.396; $\mathrm{P}<0.001)$, and age $(\mathrm{HR}, 1.528 ; 95 \% \mathrm{Cl} 0.980-2.382 ; \mathrm{P}=0.061)$. The discriminative ability and calibration of the nomograms revealed good predictive ability as indicated by the $C$ statistics $(0.715$ for DFS and 0.731 for OS).

Conclusion: Independent predictors of survival and relapse risk after IA regimen for AML can be utilized to obtain survival nomograms. These nomograms were able to predict DFS and OS while having good calibration accuracy and discriminative ability on internal validation.

\section{Background}

Acute myeloid leukemia(AML) is the most common type of acute leukemia, with the estimated 19,940 new cases and 11,180 deaths in the United States in 2020[1]. For patients with non-M3 [acute promyelocytic leukemia(APL)] de novo AML, IA regimen [idarubicin(IDA) and cytarabine(Ara-C)] as induction therapy remains the best intervention for long-term survival.[2, 3] Unfortunately, AML is a highly lethal malignancy, with up to $39-49 \%$ of patients suffering from relapse at 5-year.[4-6] Furthermore, 5-year overall survival (OS) for patients with non-M3 AML range from $24 \%$ to $40 \% .[7-10]$ 
Currently, several factors which can aid with prognostication and prediction of clinical outcomes [e.g., risk stratification, age, white blood cell (WBC) count, coexisting comorbidities and a history of an antecedent hematologic disorder].[2, 3, 11-17] The European Leukemia Net(ELN)[3] and National Comprehensive Cancer Network(NCCN)[2] risk stratifications are the most widely used criteria to stratify patients with AML. Although the risk stratification is an important factor for the prediction of survival, it is important to incorporate other clinical factors(e.g., age) to optimize prediction system.

Despite the presence of several clinicopathological factors used to predict prognosis, the accuracy of these prognosticative models remains a challenge. Thus, there have been various prognostication models developed recently to address this issue. Sorror et al. developed a novel AML composite model, which included cytogenetic risk, age, regimen intensity and comorbidities, to predict 1-year mortality.[11] Shouval, et al. constructed an auto-AML nomogram, which contained age and cytogenetic risk, to predict leukemia-free survival from autologous stem cell transplantation (auto-SCT).[18] Considering that these models were constructed based on different populations and outcomes, the availability of predictive models is restrictive.

Therefore, we aimed to develop and validate nomograms, which include clinicopathological factors, for estimate the probability of disease-free survival(DFS) and overall survival(OS) in patients with non-M3 AML undergoing " $3+7$ " IA regimen as induction chemotherapy.

\section{Methods}

\section{Patient Population and Data Collection}

We conducted a retrospective study, based on data collected by review of electronic medical records for 360 patients treated at the First Affiliated Hospital of Wenzhou Medical University between June 2008 to August 2019. All patients were newly diagnosed with de novo non-M3 AML. Only patients who received

conventional " $3+7$ " IA(IDA 8-10 mg/m2 at day 1-3 and Ara-C $100 \mathrm{mg} / \mathrm{m} 2$ at day $1-7)$ regimen as initial induction chemotherapy were included in the study group. Secondary AML patients with preceding hematological disorders were excluded. Patients with missing values on follow-up data were not included in the study. This study was approved by the institutional review board. No additional patient informed consent was required due to retrospective nature of this study. All data were collected and analyzed in accordance with the Declaration of Helsinki.

Variables concerning clinicopathological characteristics were reviewed, including age, sex, WBC count, hemoglobin( $(\mathrm{Hb})$, platelet(PLT), serum albumin, blasts at bone marrow(BM) and peripheral blood(PB), morphological immunophenotypic, cytogenetic and molecular features of myeloid blasts at the time of diagnosis before chemotherapy. Additionally, variables concerning subsequent therapeutic data were extracted, such as initial chemotherapy regimen, allogeneic hematopoietic stem cell transplantation(alloHSCT) treatment and remission status after treatment. De novo AML was diagnosed and classified according to FAB [19] and ELN 2017 criteria[3]. 


\section{Outcome}

The outcomes of interest in this study were OS and DFS. OS was calculated from the date of diagnosis to the death or last follow-up, and DFS was calculated from the date of complete remission(CR) to relapse, death or last follow-up. The criteria of CR included low residual blast percentages $(<5)$, morphologically normal hematopoiesis, recovery of peripheral blood cell counts to normal levels, and without extramedullary disease.[20-23] Relapse was defined as reoccurrence of high blast percentages $(\geq 5 \%)$ in BM unrelated to recovery from prior chemotherapy $[3,24]$.

\section{Statistical Analysis}

Numerical variables were reported as medians with interquartile ranges (IQRs), and categorical variables were reported as absolute and relative frequencies. Numerical predictors (e.g., WBC, Hb and PLT) were categorized by receiver operating characteristic $(\mathrm{ROC})$ curve, and the optimal cut-off was made by combination of Youden index and clinical use.

The associations of each variable with DFS and OS were first assessed by univariable cox proportional hazards regression analysis for investigating the independent risk factors. All variables associated with DFS and OS at a significant level were subsequently analyzed by stepwise multivariate analysis. Variables with a $p$ value $<0.05$ in multivariable analysis and variables with clinical importance identified by previously published articles $[3,4,11,12,14,15]$, were selected to incorporate in the nomograms to predict the probability of 1-year, 2-year and 3-year DFS and OS rates.

The nomograms were then constructed based on proportionally turning each regression coefficient in multivariate analysis to a 0 - to 100-point scale. The performance of nomograms were measured using the concordance index(C-index) as per Harrell et al.[25] To further assess discriminative ability of the model, the Kaplan-Meier curves of DFS and OS were plotted, stratified by the quartile of the total points calculated from the nomograms. The calibration of nomograms were evaluated by calibration curve, with bootstrap resampling to decrease the overfit bias. Statistical analyses was performed with $\mathrm{R}$ version 3.6.1(https://www.r-project.org/). Significance level was defined as $p$ value $<0.05$.

\section{Results}

\section{Baseline Characteristics}

A total of $360 \mathrm{AML}$ patients were enrolled in this study who baseline characteristics as listed in Table 1. The median age of the patient population was 48 years(IQR, $33-59$ years), $55 \%$ of patients were male(Table 1). The median WBC count was $18.25 \times 109 / \mathrm{L}(5.54-54.33)$. The median $\mathrm{Hb}$ level was 82.50g/L(66.00-103.25), and median PLT count was $40.50 \times 10^{9} / \mathrm{L}(19.75-86.25)$. The median percentages of blasts in PB and BM were $54.00 \%$ (21.50-78.00) and 66.00\% (45.80-83.28) respectively. The median albumin level was 35.95g/L (32.75-39.52). In total, 70(19.44\%), 195(54.17\%) and 83(23.06\%) patients 
displayed favorable, intermediate, and poor risk groups, respectively. In total, $21.39 \%$ of the patients underwent allo-HSCT.

The study was censored on January 22, 2020. The median follow-up time was 15.4 months (range,0.2132.8). By the time of censor, 65(18.06\%) patients did not reach CR after induction and re-induction chemotherapy, $138(38.33 \%)$ patients relapsed, and 226(62.78\%) patients died. Among them, 28 patients died of induction-related toxic effects, 21 patients died during re-induction chemotherapy, 15 patients died during maintenance and consolidation chemotherapy, 24 patients died of lack of response to induction, 124 patients died of disease-progression, 11 patients died of complications of HSCT and 3 patients died of other reasons(eTable). The unadjusted median DFS was 9.2 months $(95 \% \mathrm{Cl}, 7.0-14.8)$, and the unadjusted median OS was 21.2 months (95\% Cl, 16.6-27.6). The 1-year, 2-year, and 3-year DFS percentages were $47.6 \%(95 \% \mathrm{Cl}, 42.6-53.1), 36.0 \%(95 \% \mathrm{Cl}, 31.2-41.5)$, and $29.8 \%(95 \% \mathrm{Cl}, 25.2-35.3)$, respectively, while the OS percentages were $59.2 \%(95 \% \mathrm{Cl}, 54.2-64.5), 46.4 \%(95 \% \mathrm{Cl}, 41.4-52.1)$, and $37.8 \%(95 \% \mathrm{Cl}, 32.8-43.6)$, respectively(eFigure).

\section{Independent Prognostic Factors}

The results of univariate and multivariate analyses of DFS and OS are summarized in Table 2 and Table 3. The univariate and multivariate Cox proportional hazards regression modeling found: age $(\mathrm{HR}, 1.770$; $95 \% \mathrm{Cl}, 1.160-2.702 ; \mathrm{P}=0.008), \mathrm{Hb}(\mathrm{HR}, 0.634 ; 95 \% \mathrm{Cl}, 0.462-0.870 ; \mathrm{P}=0.005)$, albumin (HR, $0.473 ; 95 \% \mathrm{Cl}$, 0.363-0.615; $\mathrm{P}<0.001$ ) and cytogenetic / molecular risk group (intermediate vs. favorable: HR, 1.614; $95 \% \mathrm{Cl}, 1.128-2.309 ; \mathrm{P}=0.001$; poor vs. favorable: $\mathrm{HR}, 2.459 ; 95 \% \mathrm{Cl}, 1.645-3.676 ; \mathrm{P}<0.001$ ) at the time of diagnosis, and allo-HSCT treatment $(\mathrm{HR}, 0.341 ; 95 \% \mathrm{Cl}, 0.234-0.497 ; \mathrm{P}<0.001)$ were independent predictors for DFS. Similarly, the following 4 variables were found to be associated with OS: $\mathrm{Hb}$ (HR, $0.616 ; 95 \% \mathrm{Cl}, 0.438-0.866 ; \mathrm{P}=0.005)$, albumin $(\mathrm{HR}, 0.448 ; 95 \% \mathrm{Cl}, 0.340-0.591 ; \mathrm{P}<0.001)$, cyto/molecular risk group(intermediate vs. favorable: $\mathrm{HR}, 1.558 ; 95 \% \mathrm{Cl}, 1.068-2.275 ; \mathrm{P}=0.021$; poor vs. favorable: $\mathrm{HR}$, 2.348; 95\% Cl, 1.523-3.620; $\mathrm{P}<0.001)$, and allo-HSCT treatment(HR, 0.256; 95\% Cl, 0.166-0.396; $\mathrm{P}<0.001)$. Although the association of age and OS was not significant $(\mathrm{HR}, 1.528 ; 95 \% \mathrm{Cl} 0.980-2.382 ; \mathrm{P}=0.061)$, age was still selected as candidate for nomogram due to its clinical importance [5].

\section{Development and Validation of Nomograms}

Nomograms to predict 1-year, 2-year and 3-year DFS and OS of patients with non-M3 AML are shown in Figure 1. The nomograms were constructed based on the 5 variables described above (Table 2 and Table 3). Total points, calculated from the sum of the allocated number of points for each variable in the nomograms, were corresponding to specific prognoses at 1 year, 2 years and 3 years. For example, a 45year-old patient undergoing allo-HSCT, with an intermediate karyotype, a hemoglobin level of $94 \mathrm{~g} / \mathrm{L}$ and a serum albumin level of $33.9 \mathrm{~g} / \mathrm{L}$, would have a total of 155 points $(0$ point for age, 41 points for hemoglobin $<100 \mathrm{~g} / \mathrm{L}, 70$ points for albumin $<35 \mathrm{~g} / \mathrm{L}$, 44 points for intermediate risk group, 0 point for alloHSCT), for a predicted 1-year, 2-year and 3-year DFS of $59.3 \%, 45.1 \%$ and $37.5 \%$, respectively (Figure $1 \mathrm{~A}$ ). 
The discriminative abilities of the nomograms for DFS and OS were evaluated by C-index ( 0.715 for DFS and 0.731 for OS). To further evaluate the discriminative abilities, the Kaplan-Meier curves of DFS and OS were displayed in Figure 2. Patients with the lowest total points (quartile-4) had a worse DFS $(p<0.001$; Figure $2 A)$ and $O S(p<0.001$; Figure $2 B)$, compared to quartile-1, -2 and -3 . Specifically, the 3-year DFS proportion of quartile-1, $-2,-3$ and -4 was $57.1 \%(95 \% \mathrm{Cl}, 46.3-70.5 \%), 34.3 \%(95 \% \mathrm{Cl}, 26.1-44.9 \%), 19.4 \%$ (95\% Cl,11.3-33.4\%) and 7.0\%(95\% $\mathrm{Cl}, 3.1-15.8 \%)$, respectively(Figure $2 \mathrm{~A})$. While the 3-year OS proportion of quartile-1, $-2,-3$ and -4 was $68.6 \%(95 \% \mathrm{Cl}, 58.0-81.1 \%), 43.1 \%(95 \% \mathrm{Cl}, 34.5-53.9 \%), 28.6 \%(95 \% \mathrm{Cl}, 19.2-42.8 \%)$ and $10.14 \%(95 \% \mathrm{Cl}, 4.8-21.2 \%)$, respectively. The accuracy of models was also evaluated by bootstrap validation with 350 resamplings. The 116-sample bootstrapped calibration curves for prediction of 3-year DFS and OS graphically showed good agreement (Figure 3).

\section{Discussion}

De novo non-M3 AML patients have a high overall mortality rate, ranging from $60 \%$ to $76 \%$ at 5-year.[7-10] Accurate prognostic models for individuals are needed, not only to select appropriate treatment regimens but also to inform patients regarding their long-term outcomes (which may aid them in their goals of care decisions). Mohamed et al. developed an AML composite model, to estimate the risk of death within 1 year after initial chemotherapy. However, models to predict the outcomes over a longer period currently do not exist. In this study, we constructed 2 nomograms that predict 1-year, 2-year and 3-year DFS and OS for AML patients after initial IA regimen. Moreover, these nomograms were validated with good discriminative ability indicated by a C-index of 0.715 for DFS and 0.731 for OS. According to Figure 3 , the predicted probabilities of 3-year survival by the nomograms were similar to actual 3-year survival. Taken together, these results support that nomograms proposed in our study could predict the risk of relapse and survival for non-M3 AML patients.

In addition, nomograms proposed in our study incorporated previously reported variables found to be associated with prognosis of AML, including patient-specific and AML-specific features. A number of studies identified that increased age, poor-risk cytogenetics, anemia, hypoalbuminemia and treatment without allo-HSCT were correlated with inferior survival.[5, 8, 15, 26-30] Our findings corroborated with those results. WBC count proved as a predictor of death in newly diagnosed AML in some studies.[13, 31] However, we found that WBC count had a slight correlation with DFS $(p=0.065$; Table), likely due to widespread use of leukapheresis and hydroxyurea pre-induction chemotherapy in recent years.

While the cost and life expectancy of the AML varies with the baseline characteristics of the patient and available therapies [32-34], accurate prognostic model for patients with AML will be helpful to clinicians. For instance, as shown in Figure 1, allo-HSCT could increase 100 points for AML prediction, and correspondingly increase DFS and OS proportionally. Therefore, individualized prediction nomograms may play a role in choosing treatment regimen in the future.

Despite this, our study has some limitations. Firstly, the sample size was small due to the single-center nature of the study. There is a need to confirm the generalizability by conducting further multicenter 
studies in the future. Additionally, retrospective nature of the study and use of electronic medical records for data collection are other potentially limitations. Finally, despite the internal validation by bootstrap validation, external validation remains to be seen.

\section{Conclusions}

In this study, we identified several independent prognostic variables, including age, $\mathrm{Hb}$, albumin and cyto/molecular risk group at the time of diagnosis, and allo-HSCT treatment. Based on these associated factors, we developed nomograms to predict DFS and OS. Additionally, the nomograms displayed good calibration accuracy and discriminative ability on internal validation. Further studies are required to externally validated the nomograms for de novo non-M3 AML patients in the future which may serve as accurate prediction tools in this patient cohort.

\section{List Of Abbreviations}

AML, acute myeloid leukemia; OS, overall survival; DFS, disease-free survival; IQR, interquartile ranges; APL, acute promyelocytic leukemia; IDA, idarubicin; Ara-C, cytarabine; WBC, white blood cell; ELN, European Leukemia Net; NCCN, National Comprehensive Cancer Network; auto-SCT, autologous stem cell transplantation; $\mathrm{Hb}$, hemoglobin; PLT, platelet; $\mathrm{BM}$, bone marrow; $\mathrm{PB}$, peripheral blood; allo-HSCT, allogeneic hematopoietic stem cell transplantation; CR, complete remission; ROC; receiver operating characteristic; C-index, concordance index.

\section{Declarations}

\section{Ethics approval and consent to participate}

This study was approved by institutional review board of the First Affiliated Hospital of Wenzhou Medical University. Consent was waived by the institutional review board due to retrospective nature of this study, yet confidentialities of patients were protected.

\section{Consent for publication}

Not applicable.

\section{Availability of data and materials}

The datasets used or analyzed during the current study are available from the corresponding author on reasonable request.

\section{Competing interests}

The authors declare that they have no competing interests 


\section{Funding}

This study was supported by grants from the Wenzhou Municipal Sci-Tech Bureau's program(No. Y20180218), Medical Health Science and Technology Project of Zhejiang Provincial Health Commission(No.2019KY452), the Zhejiang Provincial Natural Science Foundation of China(LY20H080002) and the Clinical Research Fund Project of Zhejiang Medical Association(2016ZYCA30).

\section{Authors' contributions}

Na Wang performed the statistical analysis and was a major contributor in writing the manuscript. Kanchun Dai, Xianghong Jin and Bei Ge carried out the collection of data. Aakash Desai review the manuscript for clarity. Kang Yu participated in the design of the study. Weihong Lin and Haige Ye conceived of the study. And Haige Ye drafted the manuscript. All authors read and approved the final manuscript.

\section{Acknowledgements}

Not applicable.

\section{References}

1. Siegel, R.L., K.D. Miller, and A. Jemal, Cancer statistics, 2020. CA Cancer J Clin, 2020. 70(1): p. 7-30.

2. Tallman, M.S., et al., Acute Myeloid Leukemia, Version 3.2019, NCCN Clinical Practice Guidelines in Oncology. J Natl Compr Canc Netw, 2019. 17(6): p. 721-749.

3. Dohner, H., et al., Diagnosis and management of AML in adults: 2017 ELN recommendations from an international expert panel. Blood, 2017. 129(4): p. 424-447.

4. Grimwade, D., et al., The importance of diagnostic cytogenetics on outcome in AML: analysis of 1,612 patients entered into the MRC AML 10 trial. The Medical Research Council Adult and Children's Leukaemia Working Parties. Blood, 1998. 92(7): p. 2322-33.

5. Byrd, J.C., et al., Pretreatment cytogenetic abnormalities are predictive of induction success, cumulative incidence of relapse, and overall survival in adult patients with de novo acute myeloid leukemia: results from Cancer and Leukemia Group B (CALGB 8461). Blood, 2002. 100(13): p. 432536.

6. Thol, F., et al., Measurable residual disease monitoring by NGS before allogeneic hematopoietic cell transplantation in AML. Blood, 2018. 132(16): p. 1703-1713.

7. Kumar, C.C., Genetic abnormalities and challenges in the treatment of acute myeloid leukemia. Genes Cancer, 2011. 2(2): p. 95-107.

8. Miyawaki, S., et al., A randomized comparison of 4 courses of standard-dose multiagent chemotherapy versus 3 courses of high-dose cytarabine alone in postremission therapy for acute myeloid leukemia in adults: the JALSG AML201 Study. Blood, 2011. 117(8): p. 2366-72. 
9. Heinicke, T., et al., Reduced Relapse Incidence with FLAMSA-RIC Compared with Busulfan/Fludarabine for Acute Myelogenous Leukemia Patients in First or Second Complete Remission: A Study from the Acute Leukemia Working Party of the European Society for Blood and Marrow Transplantation. Biol Blood Marrow Transplant, 2018. 24(11): p. 2224-2232.

10. Walter, R.B., et al., Effect of complete remission and responses less than complete remission on survival in acute myeloid leukemia: a combined Eastern Cooperative Oncology Group, Southwest Oncology Group, and M. D. Anderson Cancer Center Study. J Clin Oncol, 2010. 28(10): p. 1766-71.

11. Sorror, M.L., et al., Development and Validation of a Novel Acute Myeloid Leukemia-Composite Model to Estimate Risks of Mortality. JAMA Oncol, 2017. 3(12): p. 1675-1682.

12. Zhang, Q., et al., Pretreatment platelet count predicts survival outcome of patients with de novo nonM3 acute myeloid leukemia. PeerJ, 2017. 5: p. e4139.

13. Zeller, B., et al., Hyperleucocytosis in paediatric acute myeloid leukaemia - the challenge of white blood cell counts above 200 x 10(9) /I. The NOPHO experience 1984-2014. Br J Haematol, 2017. 178(3): p. 448-456.

14. Wong, G.C., Hyperleukocytosis in acute myeloid leukemia patients is associated with high 30-day mortality which is not improved with leukapheresis. Ann Hematol, 2015. 94(12): p. 2067-8.

15. Juliusson, G., et al., Age and acute myeloid leukemia: real world data on decision to treat and outcomes from the Swedish Acute Leukemia Registry. Blood, 2009. 113(18): p. 4179-87.

16. Della Porta, M.G., Prognosis of secondary acute myeloid leukemia. Leuk Res, 2013. 37(8): p. 857-8.

17. Ofran, Y., M.S. Tallman, and J.M. Rowe, How I treat acute myeloid leukemia presenting with preexisting comorbidities. Blood, 2016. 128(4): p. 488-96.

18. Shouval, R., et al., Individualized prediction of leukemia-free survival after autologous stem cell transplantation in acute myeloid leukemia. Cancer, 2019. 125(20): p. 3566-3573.

19. Bennett, J.M., et al., Proposals for the classification of the acute leukaemias. French-American-British (FAB) co-operative group. Br J Haematol, 1976. 33(4): p. 451-8.

20. O'Donnell, M.R., et al., Acute Myeloid Leukemia, Version 3.2017, NCCN Clinical Practice Guidelines in Oncology. J Natl Compr Canc Netw, 2017. 15(7): p. 926-957.

21. Fey, M.F. and C. Buske, Acute myeloblastic leukaemias in adult patients: ESMO Clinical Practice Guidelines for diagnosis, treatment and follow-up. Ann Oncol, 2013. 24 Suppl 6: p. vi138-43.

22. Dohner, $\mathrm{H}$., et al., Diagnosis and management of acute myeloid leukemia in adults: recommendations from an international expert panel, on behalf of the European LeukemiaNet. Blood, 2010. 115(3): p. 453-74.

23. Wattad, M., et al., Impact of salvage regimens on response and overall survival in acute myeloid leukemia with induction failure. Leukemia, 2017. 31(6): p. 1306-1313.

24. Schlenk, R.F., et al., Impact of pretreatment characteristics and salvage strategy on outcome in patients with relapsed acute myeloid leukemia. Leukemia, 2017. 31(5): p. 1217-1220.

25. Harrell, F.E., Jr., et al., Evaluating the yield of medical tests. Jama, 1982. 247(18): p. 2543-6. 
26. Grosicki, S., et al., Assessing the efficacy of allogeneic hematopoietic stem cells transplantation (alloHSCT) by analyzing survival end points in defined groups of acute myeloid leukemia patients: a retrospective, multicenter Polish Adult Leukemia Group study. Am J Hematol, 2015. 90(10): p. 904-9.

27. DiNardo, C.D., et al., Interactions and relevance of blast percentage and treatment strategy among younger and older patients with acute myeloid leukemia (AML) and myelodysplastic syndrome (MDS). Am J Hematol, 2016. 91(2): p. 227-32.

28. Huck, A., et al., Prior cytopenia predicts worse clinical outcome in acute myeloid leukemia. Leuk Res, 2015. 39(10): p. 1034-40.

29. Wang, N., et al., Prognostic value of hypoalbuminemia at diagnosis in de novo non-M3 acute myeloid leukemia. Leuk Lymphoma, 2019: p. 1-9.

30. Filliatre-Clement, L., et al., Serum albumin or body mass index: Which prognostic factor for survival in patients with acute myeloblastic leukaemia? Hematol Oncol, 2019. 37(1): p. 80-84.

31. Greenwood, M.J., et al., Leukocyte count as a predictor of death during remission induction in acute myeloid leukemia. Leuk Lymphoma, 2006. 47(7): p. 1245-52.

32. Halpern, A.B., et al., Association of Risk Factors, Mortality, and Care Costs of Adults With Acute Myeloid Leukemia With Admission to the Intensive Care Unit. JAMA Oncol, 2017. 3(3): p. 374-381.

33. Vaughn, J.E., et al., Resource Utilization and Safety of Outpatient Management Following Intensive Induction or Salvage Chemotherapy for Acute Myeloid Leukemia or Myelodysplastic Syndrome: A Nonrandomized Clinical Comparative Analysis. JAMA Oncol, 2015. 1(8): p. 1120-7.

34. Wang, H.I., et al., Long-term medical costs and life expectancy of acute myeloid leukemia: a probabilistic decision model. Value Health, 2014. 17(2): p. 205-14.

\section{Tables}

Due to technical limitations, table 1,2,3 is only available as a download in the Supplemental Files section.

\section{Figures}

A

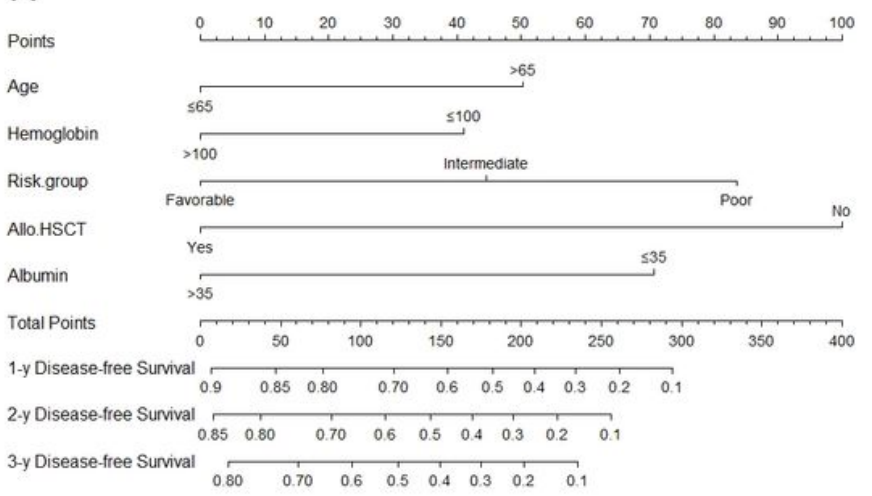

B

Points

Age

Hemoglobin

Risk.group

Allo.HSCT

Albumin

Total Points

1-y Overall Survival

2-y Overall Survival

3-y Overall Surviva

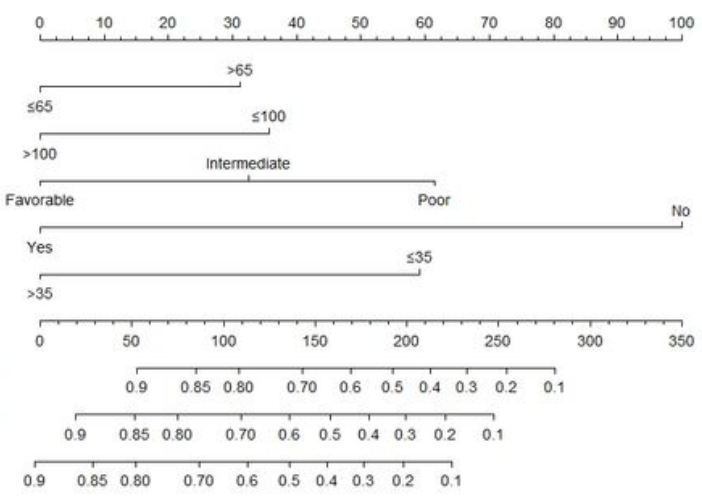


Figure 1

Nomograms predicting disease-free(A) and overall survival(B) in de novo non-M3 AML patients after IA regimen chemotherapy.
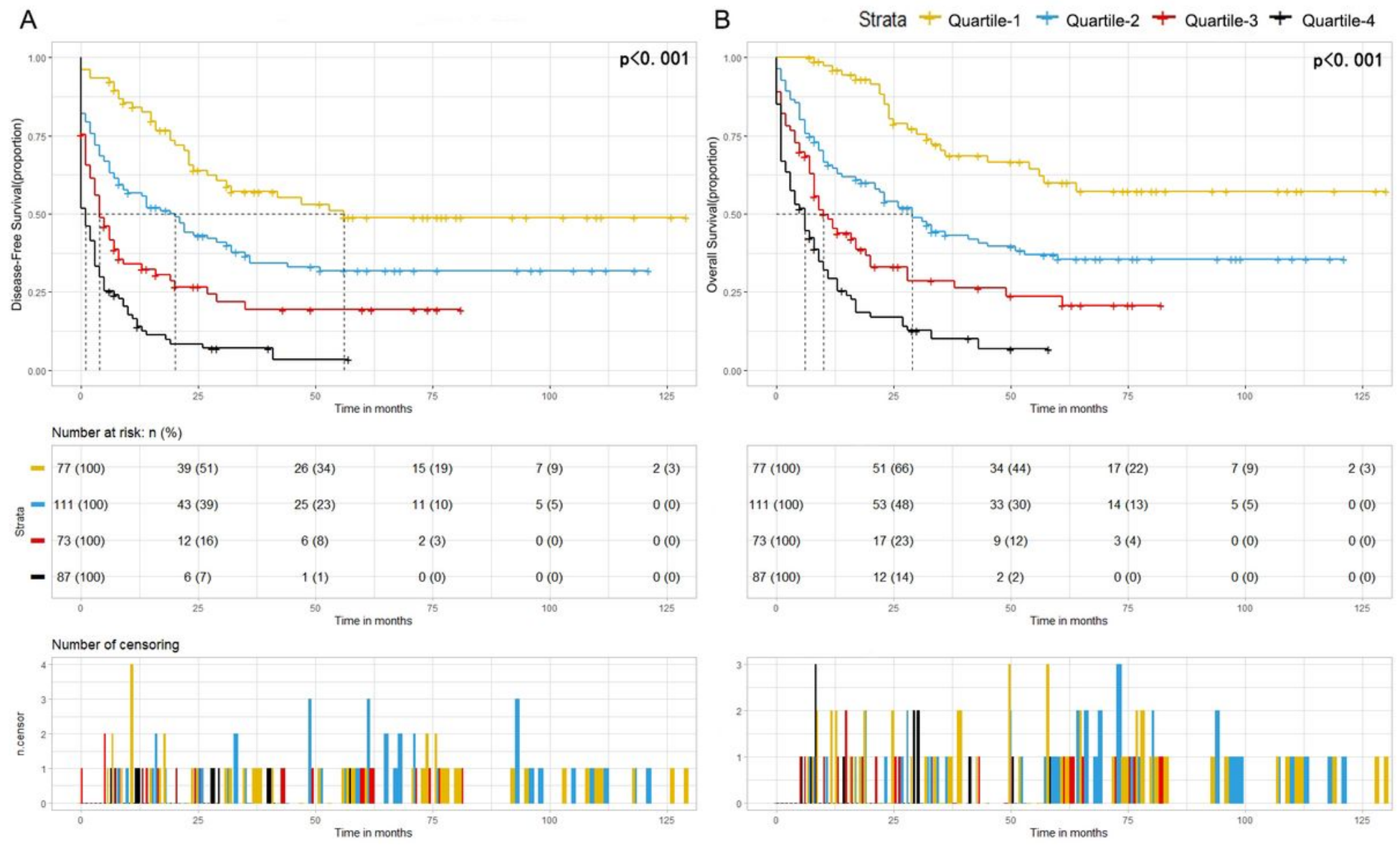

Figure 2

Kaplan-Meier curves of disease-free(A) and overall survival(B) in patients with non-M3 AML According to quartiles of total points calculated by nomograms. P-values were based on the log-rank test. 

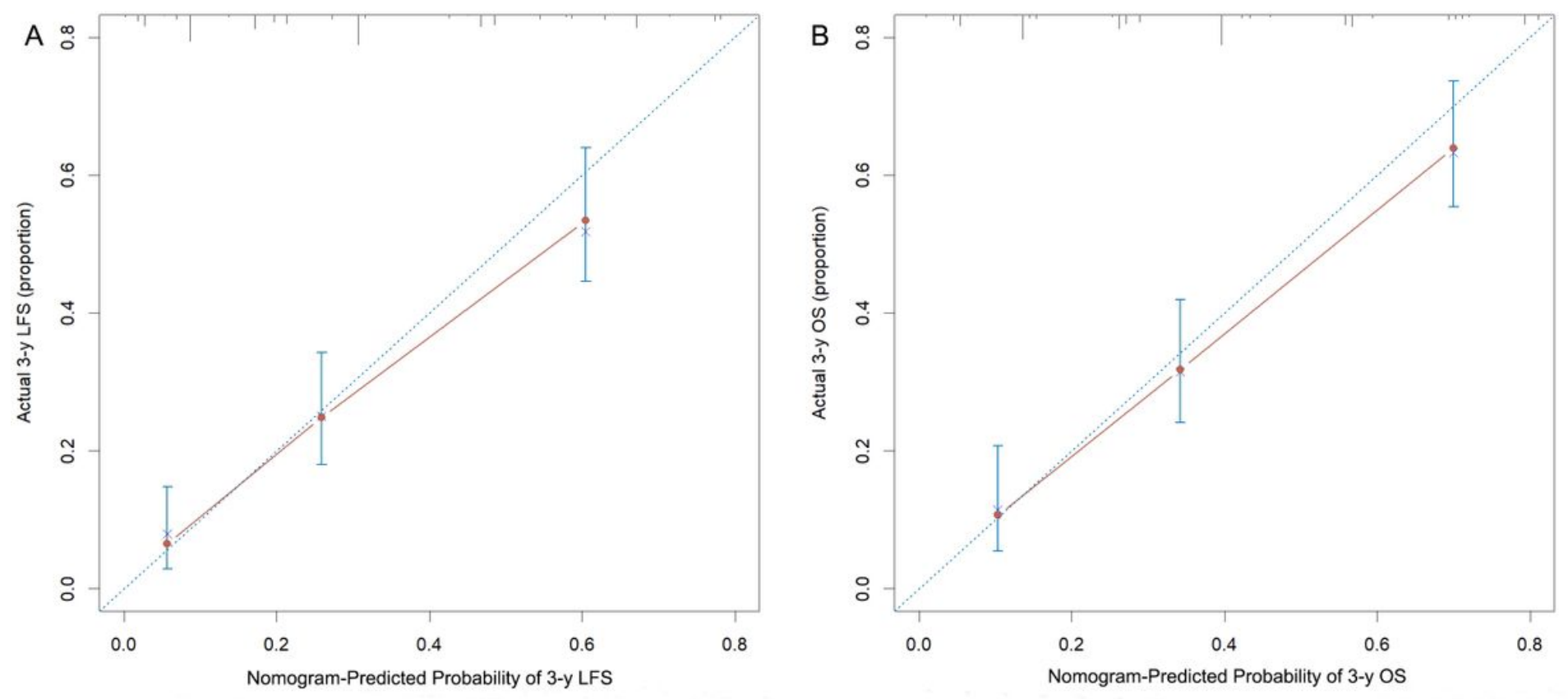

Figure 3

Calibration curve comparing predicted and actual 3-year disease-free $(A)$ and overall survival proportion(B).

\section{Supplementary Files}

This is a list of supplementary files associated with this preprint. Click to download.

- Table2.xlsx

- Table1.xlsx

- Table3.xlsx

- eFigure.jpeg

- eTable.xlsx 